\title{
Insufficienza renale acuta nello shock settico: funzione cardiaca e bilancio di ossigeno durante emofiltrazione continua
}

\author{
C. Meloni ${ }^{1}$, M. Morosetti ${ }^{2}$, G. Palombo ${ }^{2}$, F. Turani ${ }^{3}$, C. Galderisi ${ }^{2}$, L. Meschini ${ }^{4}$, \\ S. Petroni ${ }^{2}$, S. Di Giulio ${ }^{4}$, C.U. Casciani ${ }^{2}$ \\ ${ }^{I}$ Istituto Tipizzazione Tissutale, Sezione Romana, CNR, \\ ${ }^{2}$ Clinica Chirurgica e ${ }^{3}$ Clinica Anestesiologica, Università Tor Vergata - Roma \\ ${ }^{4}$ Nefrologia e Dialisi, Ospedale GB. Grassi, USL RMD - Roma
}

insufficienza renale acuta (IRA) durante multiple organ failure (MOF) è un'evenienza frequente, caratterizzata da elevata mortalità nonostante il costante miglioramento delle tecniche dialitiche e rianimatorie $(1,2)$. Il trattamento extracorporeo continuo (CRRT), comprendendo l'emofiltrazione artero-venosa continua (CAVH) (3) e le successive modifiche, rappresenta un supporto indispensabile nel paziente critico affetto da IRA (4), anche se la sua eventuale incidenza sulla prognosi di tali pazienti ha fornito dati discordanti $(5,6)$. È stato dimostrato che la CAVH migliora i parametri emodinamici dello shock settico (7), mentre i dati relativi al bilancio di ossigeno sono, al contrario, controversi $(8,9)$. Lo shock settico è caratterizzato dal punto di vista emodinamico da basse resistenze sistemiche, bassa pressione arteriosa sistemica con elevato cardiac output. Durante lo shock settico possono coesistere ipertensione arteriosa polmonare e danno ventricolare destro con netto peggioramento della prognosi $(10,11)$. Nello shock settico c'è inoltre discrepanza tra la richiesta sistemica e la disponibilità di ossigeno. La terapia dello shock settico consiste nel ripristinare e mantenere un'adeguata perfusione d'organo mediante infusione di liquidi per raggiungere un volume intravascolare ottimale. Un sovraccarico di liquidi, però, può determinare edema interstiziale con effetti negativi sulla ossigenazione tissutale e, quindi, produrre danno d'organo (12). Aumentati livelli di consumo di ossigeno sono associati ad un migliore outcome dalla sepsi severa (13). Scopo del nostro studio è stato quello di correlare i parametri emodinamici, ossimetrici e metabolici di un gruppo di pazienti settici durante il trattamento con CRRT al fine di verificare se le modificazioni dei parametri emodinamici influenzano il bilancio di ossigeno. Inoltre, utilizzando due metodiche diverse, abbiamo voluto studiare il differente impatto delle due tecniche sulla funzione cardiaca e sul bilancio di $\mathrm{O}_{2}$.

\section{Pazienti e metodi}

Ventinove pazienti affetti da shock settico $(19$ maschi, 10 femmine, età media $48.0 \pm 17.6$ anni, range 28 63 anni) sono stati studiati presso il reparto di Rianimazione dell'Ospedale S. Eugenio, Università Tor Vergata di Roma. Le cause della sepsi erano: politraumatismo ( 7 casi), pancreatite acuta necrotico-emorragica ( 6 casi), ustioni estese ( 3 casi), infezioni polmonari in pazienti trapiantati (5 casi), peritoniti (3 casi), leucemie acute (3 casi). La sepsi fu diagnosticata secondo i criteri di Bone (14).

Lo shock fu valutato dalla presenza di uno dei seguenti segni: pressione sistolica intra-arteriosa $<90 \mathrm{mmHg}$ per più di una ora; infusione endovenosa di catecolamine per più di un'ora al fine di mantenere una pressione sistolica intra-arteriosa $\geq 90 \mathrm{mmHg}$; lattati $>3.0 \mathrm{mmol} / \mathrm{L}$. Tutti i pazienti erano intubati e ventilati meccanicamente sia in ventilazione meccanica controllata che in ventilazione sin- 
cronizzata intermittente, mantenendo la $\mathrm{PCO}_{2}$ tra 35 e $45 \mathrm{mmHg}$, la $\mathrm{PO}_{2}$ $>85 \mathrm{mmHg}$ e la $\mathrm{SaO}_{2}>91 \%$. In tutti i pazienti le condizioni emodinamiche venivano mantenute mediante la infusione di dopamina $(6 \mathrm{mcg} / \mathrm{kg} / \mathrm{min})$ durante il periodo dello studio. Ogni paziente era monitorizzato con un catetere di Swan-Ganz modificato (Thermodilution Ejection Fraction/ Volumetric Catheter modello 93A754H-7.5 F, Baxter Health Care Corporation), posizionato nell'arteria polmonare attraverso la vena giugulare interna. Un catetere arterioso era usato per monitorizzare la pressione arteriosa e la frequenza cardiaca (Siemens Sirecust mod. 402). In tutti i pazienti venivano monitorizzati i seguenti parametri: pressione arteriosa media (MAP), frequenza cardiaca (HR), pressione arteriosa polmonare (PAP), pressione polmonare capillare di incuneamento (PAWP), pressione venosa centrale (CVP), cardiac output $(\mathrm{CO})$, frazione d'eiezione ventricolo destro (RVEF), end-systolic volume ventricolo destro (RVESV), end-diastolic volume ventricolo destro (RVEDV), saturazione di ossigeno del sangue venoso misto $\left(\mathrm{PvO}_{2}\right)$. $\mathrm{CO}$ e RVEF venivano determinati sulla media di tre determinazioni successive. I seguenti parametri venivano calcolati mediante formule matematiche: cardiac index $(\mathrm{CI})$, indice dell'end-systolic volume ventricolo destro (RVESVI), indice dell'enddiastolic volume ventricolo destro, indice delle resistenze vascolari sistemiche (SVRI), rapporto di estrazione di $\mathrm{O}_{2}\left(\mathrm{O}_{2} \mathrm{ER}\right)$, indice di disponibilità di ossigeno $\left(\mathrm{DO}_{2} \mathrm{I}\right)$, indice di consumo di ossigeno $\left(\mathrm{VO}_{2} \mathrm{I}\right)$. I parametri emodinamici, biochimici e $\mathrm{i}$ dati relativi all' $\mathrm{O}_{2}$ venivano rilevati all' inizio del trattamento (t0), dopo $24(\mathrm{tl})$ e 48 (t2) ore. Tutti i pazienti erano alimentati mediante nutrizione parenterale totale $(2000-3000 \mathrm{cc} / 24$ ore $)$ già prima dell'inizio del trattamento con emofiltrazione continua.

Tutti i pazienti erano affetti da IRA oligoanurica (diuresi media / 24 ore: $277 \pm 36 \mathrm{cc}$, range $0-350 \mathrm{cc}$ ) ed avevano un notenole sovraccarico idrico. Dei 29 pazienti, 18 (11 maschi, 7 femmine, età media $46.6 \pm$
15.0 anni, range $30-61$ anni) (GRUPPO A) venivano trattati con CAVH. Questa tecnica veniva eseguita usando un filtro capillare in poliacrilonitrile da $0.43 \mathrm{~m}^{2}$ connesso a due cateteri a lume singolo in arteria e vena femorale. I filtri venivano sostituiti ogni 24 ore oppure se coagulati $o$ in caso di funzione non adeguata (ultrafiltratione $<250 \mathrm{ml} / \mathrm{h}$ per 3 ore consecutive). Il flusso medio di ultrafiltrazione era di $568 \pm 125 \mathrm{ml} / \mathrm{h}$. Come liquido di reinfusione veniva usata una soluzione in bicarbonato (Biosol 20, Hospal Dasco, Francia).

Undici pazienti (GRUPPO B) (8 maschi, 3 femmine, età media $50.1 \pm$ 18.3 anni, range $28-63$ anni) venivano trattati con emodiafiltrazione continua (CAVHD) (5) per evitare uno stato di ipercatabolismo (15). La CAVHD veniva effettuata usando un filtro capillare in poliacrilonitrile da $0.43 \mathrm{~m}^{2}$ connesso mediante due cateteri a lume singolo ad arteria e vena femorale. Il dialisato (Biosol 20, Hospal Dasco, France) veniva infuso ad $1 \mathrm{~L} / \mathrm{h}$. Il flusso di ultrafiltrazione era di $349 \pm 81 \mathrm{ml} / \mathrm{h}$.

Emogasanalisi, azotemia, creatinina, sodio, potassio, calcio, fosforo, ematocrito, emoglobina e lattati erano valutati ogni 24 ore di trattamento con CAVH e CAVHD.

Il valore medio di Apache II scores (16) era di $29.1 \pm 4.0$ (range $25-41$ ), comparabile nei 2 gruppi: $28.8 \pm 4.9$ (range 26-39) nel GRUPPO A e 29.6 \pm 3.7 (range 25-41) nel GRUPPO B.

I dati sono espressi come media \pm deviazione standard. Il test ANOVA è stato usato per valutare le variazioni nel tempo; lo Student t test è stato usato per confrontare le differenze tra i 2 gruppi. Un $\mathrm{p}<0.05$ è stato considerato significativo.

\section{Risultati}

In Tabella I sono riportati i dati riguardanti la funzione cardiaca e il bilancio di $\mathrm{O}_{2}$ di tutti i 29 pazienti durante trattamento con emofiltrazione continua. CVP e PAWP diminuivano significativamente già 24 ore dopo l'inizio del trattamento con CAVH(D) (rispettivamente
CVP $22.3 \pm 1.7 \mathrm{cmH}_{2} \mathrm{O}$ vs $18.2 \pm$ $0.9, \mathrm{p}<0.05$; PAWP $20.6 \pm 7.4$ $\mathrm{mmHg}$ vs $16.3 \pm 5.0 \mathrm{mmHg}$, $\mathrm{p}<0.05)$. Allo stesso tempo RVEDVI diminuiva significativamente $\left(136 \pm 29\right.$ vs $67 \pm 23 \mathrm{ml} / \mathrm{min} / \mathrm{m}^{2}$ dopo 24 ore, $\mathrm{p}<0.05)$. Al contrario, RVEF migliorava significativamente solo dopo 48 ore di trattamento con CAVH(D) e c'era una riduzione del CI associato ad un incremento del SVRI. I dati relativi al monitoraggio dell' $\mathrm{O}_{2}$ non si modificavano durante tutto il trattamento. I lattati diminuivano in maniera significativa durante tutto il trattamento (da $5.9 \pm 1.9 \mathrm{mmol} / \mathrm{L}$ all'inizio a $3.7 \pm$ 0.4 dopo 24 ore di trattamento, $\mathrm{p}$ $<0.05$, e $2.7 \pm 0.3 \mathrm{mmol} / \mathrm{L}$ dopo 48 ore, $\mathrm{p}<0.01)$.

Come dimostra la Tabella II, l'emofiltratione continua risulta ottimale per la rimozione di liquidi, il bilancio elettrolitico e il controllo metabolico in tutti i pazienti oggetto dello studio. La tecnica è stata ben tollerata nonostante le gravi condizioni emodinamiche dei pazienti, con MAP ed HR che non subivano variazioni durante tutto il trattamento.

I parametri relativi alla funzione cardiaca ed al bilancio di $\mathrm{O}_{2}$ sono stati confrontati nelle due tecniche usate. Nessuna differenza veniva rilevata sui parametri relativi al consumo di $\mathrm{O}_{2}$, mentre differenze significative si notavano dopo 24 ore di trattamento nei parametri relativi alla rimozione di liquidi. Infatti erano significativamente diverse dopo 24 ore CVP $\left(17.3 \pm 4.2 \mathrm{cmH}_{2} \mathrm{O}\right.$ durante CAVH vs $20.1 \pm 3.8$ durante CAVHD, $\mathrm{p}<0.05$ ) e PAWP $(16.4 \pm 6.7$ durante CAVH vs $18.0 \pm 7.4$ durante CAVHD, $\mathrm{p}<0.05$ ). In Tabella III sono riportati i dati relativi a 48 ore di trattamento con le due tecniche: nessuna differenza significativa tra i parametri studiati veniva evidenziata.

La durata media del trattamento è stata di $17.5 \pm 5.3$ giorni (range 8 29 giorni) senza differenza tra le due tecniche. La sopravvivenza media è stata del $27.5 \%$, comparabile nei 2 gruppi, rispettivamente $5 / 18(27.7 \%)$ nel gruppo A e 3/11 (27.2\%) nel gruppo B. Durante il periodo di studio non si sono verificati decessi. 
TABELLA I - PARAMETRI EMODINAMICI E BILANCIO DI OSSIGENO ALL'INIZIO DELLO STUDIO (T0) E DOPO 24 (T1) AND 48 (T2) ORE IN 29 PAZIENTI SETTICI TRATTATI CON EMOFILTRAZIONE CONTINUA

\begin{tabular}{lccc}
\hline & \multicolumn{1}{c}{ T0 } & T1 & T2 \\
\hline HR & $101 \pm 19$ & $104 \pm 18$ & $100 \pm 16$ \\
MAP & $79 \pm 17$ & $81 \pm 21$ & $77 \pm 9.6$ \\
CVP & $21.3 \pm 2.1$ & $18.2 \pm 0.9^{\circ}$ & $16.7 \pm 1.1^{*}$ \\
PAWP & $20.6 \pm 6.9$ & $17.3 \pm 5.0^{\circ}$ & $13.1 \pm 5.8^{*}$ \\
CI & $5.4 \pm 2.2$ & $4.8 \pm 1.1^{\circ}$ & $4.3 \pm 1.2^{*}$ \\
RVEF & $25.1 \pm 5.6$ & $30.9 \pm 6.1$ & $33.9 \pm 6.5^{*}$ \\
SVRI & $1143 \pm 488$ & $1371 \pm 621$ & $1500 \pm 474^{*}$ \\
REDVI & $139 \pm 29$ & $67 \pm 23^{\circ}$ & $58 \pm 24^{*}$ \\
$\mathrm{O}_{2}$ ER & $0.29 \pm 0.1$ & $0.30 \pm 0.1$ & $0.31 \pm 0.1$ \\
PVO $_{2}$ & $40 \pm 8.0$ & $38 \pm 4.1$ & $39 \pm 4.6$ \\
DO $_{2} 1$ & $578 \pm 235$ & $549 \pm 230$ & $497 \pm 193$ \\
VO $_{2} \mathrm{I}$ & $190 \pm 79$ & $161 \pm 54$ & $157 \pm 44$ \\
LATTATI $^{\circ}$ & $5.9 \pm 1.9$ & $3.7 \pm 0.2^{\circ}$ & $2.7 \pm 0.3^{*}$ \\
\hline
\end{tabular}

${ }^{\circ} p<0.05 T 1$ vs T0; ${ }^{*} p<0.05 T 2$ vs T0.

HR: b/min; MAP: mmHg; CVP: $\mathrm{cmH}_{2} \mathrm{O}$; PAWP: $\mathrm{cmH}_{2} \mathrm{O} ; \mathrm{CI}: \mathrm{L} / \mathrm{min} / \mathrm{m}^{2} ; \mathrm{RVEF}: \%$; SVRI: dyne.sec/cm-5; REDVI: $\mathrm{ml} / \mathrm{min} / \mathrm{m}^{2} ; \mathrm{O}_{2} E R: \% ; \mathrm{PVO}_{2}: \mathrm{mmHg} ; \mathrm{DO}_{2} I: \mathrm{ml} / \mathrm{min} / \mathrm{m}^{2}$; $\mathrm{VO}_{2} \mathrm{I}: \mathrm{ml} / \mathrm{min} / \mathrm{m}^{2}$; Lattati: $\mathrm{mmol} / \mathrm{L}$

TABELLA II - PARAMETRI BIOCHIMICI IN 29 PAZIENTI SETTICI CON IRA PRIMA (T0) E DOPO 48 ORE (T2) DI TRATTAMENTO CON EMOFILTRAZIONE CONTINUA

\begin{tabular}{llcc}
\hline & & T0 & \multicolumn{1}{c}{ T2 } \\
\hline BUN & $(\mathrm{mg} / \mathrm{dl})$ & $86.7 \pm 18.9$ & $60.3 \pm 14.3$ \\
Creatinina & $(\mathrm{mg} / \mathrm{dl})$ & $4.5 \pm 1.2$ & $2.8 \pm 1.0$ \\
Sodio & $(\mathrm{mEq} / \mathrm{L})$ & $132.8 \pm 10.6$ & $140.6 \pm 6.9$ \\
Potassio & $(\mathrm{mEq} / \mathrm{L})$ & $5.0 \pm 1.4$ & $4.0 \pm 0.7$ \\
Calcio & $(\mathrm{mg} / \mathrm{dl})$ & $8.5 \pm 1.3$ & $8.7 \pm 0.6$ \\
Fosfato & $(\mathrm{mg} / \mathrm{dl})$ & $5.3 \pm 1.1$ & $5.1 \pm 0.8$ \\
$\mathrm{Ht}$ & $(\%)$ & $30.1 \pm 5.0$ & $31.5 \pm 3.3$ \\
$\mathrm{Hb}$ & $(\mathrm{g} / \mathrm{L})$ & $9.4 \pm 1.9$ & $10.2 \pm 1.8$ \\
$\mathrm{PLT}$ & $\left(\mathrm{count} / \mathrm{m}^{3}\right)$ & $283 \pm 77$ & $250 \pm 65$ \\
$\mathrm{pH}$ & & $7.38 \pm 0.02$ & $7.42 \pm 0.02$ \\
$\mathrm{HCO}-$ & $(\mathrm{mmol} / \mathrm{L})$ & $20.8 \pm 1.2$ & $23.6 \pm 0.6$ \\
Delta weight & $(\mathrm{kg} / 24 \mathrm{~h})$ & & $2.3 \pm 0.5$ \\
\hline
\end{tabular}

ne continua. La normalizzazione del cardiac index può essere correlata ad un decremento della risposta iperdinamica, così come durante lo studio le SVRI aumentano, mentre il rapporto $\mathrm{VO}_{2} / \mathrm{DO}_{2}$ si mantiene nel range fisiologico. La riduzione del CI durante CAVH (D) non è da mettere in relazione con eventuale accumulo dei lattati, che possono avere effetti negativi sul cardiac output (25). Infatti i lattati diminuiscono durante il trattamento e questo dato può rappresentare un marker indiretto di migliorata perfusione d'organo, confermata dall'aumento delle resistenze sistemiche periferiche. Inoltre, i nostri dati dimostrano che la CAVH (D) induce una significativa riduzione delle pressioni di riempimento (REDVI and PCWP) e sembra attenuare la risposta iperdinamica, come dimostrato dalla concomitante riduzione del $\mathrm{CI}$ e miglioramneto del SVRI. La riduzione del fluid over-

\section{Discussione}

La sepsi e lo shock settico, definite come sindromi ipermetaboliche con aumentata richiesta di ossigeno (17), rappresentano attualmente una delle maggiori cause di morbidità e mortalità nei paesi industrializzati (18).

L'emofiltrazione continua è la terapia sostitutiva di scelta nei pazienti critici ed in particolare in quelli con multiple organ failure $(19,20)$. Tale tecnica, secondo alcuni Autori, può rappresentare un nuovo approccio terapeutico in numerose malattie come sepsi/shock settico (systemic inflammatory response syndrome - SIRS), addirittura in assenza di alterazioni renali (21). Tuttavia anche i trattamenti extracorporei continui sono associati ad effetti positivi e negativi. Tra gli effetti positivi possiamo considerare la possibilità di ripristinare un giusto peso corporeo, specialmente a carico del volume extra cellulare, buona stabilità emodinamica (22), riduzione della temperatura corporea, rimozione di mediatori dell'infiammazione, infusione di tamponi organici che possono avere effetti importanti sul metabolismo e sulla funzione cardiaca. Tra gli effetti negativi consideriamo la protratta interazione sangue-membrana che può incidere negativamente sul catabolismo proteico ed aggravare la SIRS (23). Inoltre, il decremento di liberazione di $\mathrm{O}_{2}$ può favorire lo sviluppo di un deficit regionale di ossigeno.

I nostri dati confermano che l'emofiltratione continua può essere utile nei pazienti settici per trattare l'IRA e il sovraccarico idrico senza incidere in modo significativo sulla stabilità emodinamica. In particolare i nostri dati mostrano che durante il trattamento RVEF e RVEDVI migliorano progressivamente e le pressioni di riempimento diminuiscono verso i valori normali senza effetti avversi sul bilancio di $\mathrm{O}_{2}$. La rimozione di liquidi mediante ultrafiltrazione riduce l'effettivo volume circolante, portando ad una riduzione del cardiac output e della disponibilità di ossigenazione cerebrale di $\mathrm{O}_{2}$ (24). Inoltre i nostri dati mostrano anche una significativa riduzione del CI dopo 48 ore di trattamento con emofiltrazio- 
TABELLA III - PARAMETRI EMODINAMICI E BILANCIO DI O ${ }_{2}$ ALL'INIZIO DELLO STUDIO (T0) E DOPO 48 ORE (T2) IN 8 PAZIENTI SETTICI TRATTATI CON CAVH (GRUPPO A) E 11 TRATTATI CON CAVHD (GRUPPO B)

\begin{tabular}{ccccc}
\hline \multicolumn{2}{c}{ GRUPPO A } & \multicolumn{2}{c}{ GRUPPO B } \\
\hline T0 & T2 & & T0 & T2 \\
\hline $102 \pm 15$ & $104 \pm 13$ & HR & $98 \pm 21$ & $96 \pm 18$ \\
$80 \pm 19$ & $79 \pm 9.3$ & MAP & $77 \pm 15$ & $74 \pm 10$ \\
$23.2 \pm 2.4^{*}$ & $17.9 \pm 2.0$ & CVP & $21 \pm 2.7^{*}$ & $18.2 \pm 1.9$ \\
$22.1 \pm 6.4^{*}$ & $12.9 \pm 6.7$ & PAWP & $19.6 \pm 7.4^{*}$ & $13.3 \pm 5.0$ \\
$5.5 \pm 2.3^{*}$ & $4.2 \pm 1.1$ & CI & $5.1 \pm 2.2^{*}$ & $4.5 \pm 1.3$ \\
$24.4 \pm 4.3^{*}$ & $34.1 \pm 6.0$ & RVEF & $26.1 \pm 6.7^{*}$ & $31.9 \pm 6.8$ \\
$1137 \pm 525^{*}$ & $1507 \pm 310$ & SVRI & $1153 \pm 433^{*}$ & $1471 \pm 521$ \\
$143 \pm 34^{*}$ & $59 \pm 26$ & REDVI & $136 \pm 25^{*}$ & $57 \pm 23$ \\
$0.30 \pm 0.1$ & $0.31 \pm 0.1$ & $\mathrm{O}_{2}$ ER & $0.29 \pm 0.1$ & $0.31 \pm 0.1$ \\
$39 \pm 7.2$ & $39 \pm 5.4$ & PVO & $41 \pm 8.4$ & $39 \pm 4.1$ \\
$570 \pm 201$ & $465 \pm 147$ & DO $_{2}$ I & $588 \pm 274$ & $509 \pm 210$ \\
$194 \pm 82$ & $154 \pm 35$ & VO $_{2}$ I & $170 \pm 79$ & $164 \pm 54$ \\
$6.2 \pm 1.7^{*}$ & $2.6 \pm 0.5$ & LATTATI $^{*}$ & $5.9 \pm 1.9^{*}$ & $3.0 \pm 0.2$
\end{tabular}

${ }^{*} p<0.05 T 2$ vs $T 0$

$T 2$ A vs T2 B: non significativo

HR: b/min; MAP: mmHg; CVP: $\mathrm{cmH}_{2} \mathrm{O}$; PAWP: $\mathrm{cmH}_{2} \mathrm{O} ; \mathrm{CI}: \mathrm{L} / \mathrm{min} / \mathrm{m}^{2} ; \mathrm{RVEF}$ : \%; SVRI: dyne.sec/cm-5; REDVI: $\mathrm{ml} / \mathrm{min} / \mathrm{m}^{2} ; \mathrm{O}_{2} E R$ : $\% ; \mathrm{PVO}_{2}: \mathrm{mmHg} ; \mathrm{DO}_{2} \mathrm{I}: \mathrm{ml} / \mathrm{min} / \mathrm{m}^{2} ; \mathrm{VO}_{2} \mathrm{I}: \mathrm{ml} / \mathrm{min} / \mathrm{m}^{2} ;$ Lattati: $\mathrm{mmol} / \mathrm{L}$

load e della concentrazione plasmatica dei lattati porta ad un miglioramento della performance cardiaca e del rapporto $\mathrm{PaO}_{2} / \mathrm{FiO}_{2}$. La rimozione di liquidi in eccesso può essere ottenuta anche mediante il trattamento emodialitico intermittente, ma quest'ultimo comporta una rapida riduzione del cardiac output associata ad abbassamento della MAP, della $\mathrm{DO}_{2}$ tissutale e della capacità estrattiva tissutale di $\mathrm{O}_{2}$, senza incidere significativamente sulle resistenze vascolari sistemiche $(20,26)$. I nostri dati sono stati focalizzati sul ruolo della rimozione dei liquidi sulla performance cardiaca, mentre la rimozione dei mediatori della sepsi (TNF, IL-6, IL-1 B, filterable cardiodepressant factor in sepsis) (7) non è stata presa in considerazione. Peraltro rimane controverso il ruolo delle citochine sull'emodinamica in corso di sepsi, mentre dati discordanti riguardo la loro rimozione mediante emofiltratione continua sono stati riportati anche recentemente (27-29). Il consumo di $\mathrm{O}_{2}$ non subisce variazioni durante le 48 ore di osservazione, mentre, al contrario, veniva notato un miglioramento sebbene non significativo dell' $\mathrm{O}_{2} \mathrm{ER}$, che può essere considerato come un indice di capacità estrattiva tissutale di $\mathrm{O}_{2}$. I nostri dati mostrano che un adeguato bilancio di $\mathrm{O}_{2}$ non è influenzato dal trattamento di emofiltrazione continua.

L'indice di mortalità rimane elevato nonostante il miglioramento della funzione cardiaca e la stabilità del bilancio di ossigeno. È comunque importante sottolineare l'elevato Apache score dei pazienti oggetto del nostro studio. Inoltre, durante il periodo di osservazione di 48 ore non avveniva alcun decesso. Si può supporre che l'automantenimento di una reazione a cascata e l'interazione con i mediatori dell'infiammazione, partendo dall'attivazione del complemento, definita MOF (30) supera a lungo termine gli effetti positivi dell'emofiltrazione continua.

In conclusione l'emofiltrazione continua può essere utile nei pazienti settici per il trattamento dell'IRA e del sovraccarico idrico. Già dopo 24 ore di trattamento la funzione ventricolare destra migliora, le pressioni di riempimento diminuiscono significativamente così come la risposta iper- dinamica con diminuzione del CI ed aumento del SVRI. Durante il periodo di studio il consumo di ossigeno non si è modificato. È comunque consigliabile correggere il sovraccarico idrico progressivamente per evitare una eccessiva riduzione del volume circolante effettivo provocando un ridotto cardiac output e ridotta disponibilità di $\mathrm{O}_{2}$ per gli organi vitali.

\section{BIBLIOGRAFIA}

1.

Spiegel DM, Ullian ME, Zerbe GO, Berl T. Determinant of survival and recovery in acute renal failure dialyzed in intensive care units. Am J Nephrol 1991; 11: 44-7.

2. Biesenbach G, Zazgornik J, Kaiser W, Grafinger P, Stuby U, Necek S. Improvement in prognosis of patients with acute renal failure over a period of 15 years: An analysis of 710 cases in a dialysis center. Am J Nephrol 1992; 12: 319-25.

3. Kramer P, Wigger W, Rieger 
J, Matthaei D, Scheler F. Arteriovenous haemofiltration: A new and simple method for treatment of overhydrated patients resistent to diuretics. Klin Wochenschr 1977; 55: 1121-2.

4. Bellomo R, Parkin G, Love J, Boyce N. Use of continuous hemofiltration: An approach to the management of acute renal failure in the critically ill. Am J Nephrol 1992; 12: 240-5.

5. Bellomo R, Farmer M, Boyce N. A prospective study of continuous hemofiltration in the management of severe acute renal failure in critically ill surgical patients. Renal Failure 1994;16:759-66.

6. Meloni C, Morosetti M, Turani F, Pet al. Cardiac function and oxygen balance in septic patients during continuous hemofiltration. Blood Purif 1998; 16: 140-6.

7. Meloni C, Morosetti M, Turani $\mathrm{F}$, et al. Monitoring of left and right ventricular function during continuous hemofiltration in critically ill patients. In: Timio M, Wizmann V eds. Cardionephrology 3. Bios ed., Cosenza, 1995; 503-5.

8. Cosentino F, Paganini E, Lockrem J, Stoller J. Continuous arteriovenous hemofiltration in the adult respiratory distress syndrome. A randomized trial. In: Sieberth $\mathrm{HG}$, Mann H, Stummvol HK, eds. Continuous Hemofiltration. Contrib Nephrol, Basel: Karger, 1991; 93: 94-7.

9. Druml W. Nonrenal indications for continuous hemofiltration therapy in patients with normal renal function? Contrib Nephrol 1995; 116: 121-9.

10. Redl G, Germann P, Plattner H, Hammerle A. Right ventricular function in early septic shock states. Intensive Care Med 1993; 19: 3-7.

11. Jardin F, Brunney D, Auvert B. Sepsis-related cardiogenic shock. Crit Care Med 1990; 18: 1055-62.

12. Chang N, Goodson WH, Gottrup F, Hunt TK. Direct measurement of wound and tissue oxygen tension in postoperative patients. Ann Surg 1983;197:470-8.

13. Tuchschmidt J, Fried J, Swinney R, Sharma O. Early hemodynamic correlates of survival in patients with septic shock. Crit Care Med 1989; 17: 719-23.

14. Bone RC, Fisher CJ, Clemmer TP, Slotman GJ, Metz CA, Balk RA. Sepsis syndrome: a valid clinical entity. Crit Care Med 1989; 17: 389-93.

15. Bonnardeaux A, Pichette V, Ouimet D, Geadah D, Habel F, Cardinal J. Solute clearance with high dialysate flow rates and glucose absorption from the dialysate in continuous arteriovenous hemodialysis. Am J Kidney Dis 1992; 19: 31-3

16. Knaus WA, Draper EA, Douglas PW, Zimmerman JE. A severity of disease classification system. Crit Care Med 1985; 13: 818-29.

17. Tuchschmidt J, Fried J, Astiz M, Rackow E. Elevation of cardiac output and oxygen delivery improves outcome in septic shock. Chest 1992; 102: 216-20.

18. Suter PM, Pittet D. Prognostic importance of vital organ dysfunction in sepsis. In: Sieberth HG, Stummvol HK, Kierdorf H, eds. Continuous extracorporeal treatment in multiple organ dysfunction. Contrib Nephrol, Basel: Karger, 1996; 116: 16-21.

19. Price CA. Continuous renal replacement therapy: the treatment of choice for acute renal failure. Ann J 1991; 18: 239-44.

20. Davenport A, Will EJ, Davidson AM. Improved cardiovascular stability during continuous modes of renal replacement therapy in critically ill patients with acute hepatic and renal failure. Crit Care Med 1993; 21: 328-38.

21. Hoffman JN, Hartl WH, Deppisch R, Faist E, Jochum M, Inthorn D. Effect of hemofiltration of hemodynamics and systemic concentration of anaphylatoxin and cytokines in human sepsis. Intensive Care Med 1996; 22: 1360-7.
22. Meloni C, Morosetti M, Meschini L, et al. Blood purification procedures for acute renal failure: convenient strategy related to clinical conditions. Blood Purif 1996; 14: $242-8$.

23. Hakim RM. Clinical implications of hemodialysis membrane biocompatibility. Kidney Int 1993; 44: 484-94.

24. Davenport A, Kirby SA. Intensive care management of patients with acute hepatic and renal failure. In: Sieberth HG, Stumm voll HK, Kierdorph, eds. Continuous treatment in multiple organ dysfunction syndrome. Contrib Nephrol, Basel: Karger, 1995; 116: 22-7.

25. Mizock BA, Falk JL. Lactic acidosis in critical illness. Crit Care Med 1992; 20: 80-93.

26. van Bommel EFM, Bouvy ND, So KL, et al. Acute dialytic support for the critically ill: Intermittent hemodialysis versus continuous arteriovenous hemodiafiltration. Am J Nephrol 1995; 15: 192-200.

27. Sanchez-Izquierdo Riera JA, Perez Vela JL, Lozano Quintana MJ, Alted Lopez E, Ortuno de Solo B, Ambros Checa A. Cytokine clearance during venovenous hemofiltration in the trauma patient. Am J Kidney Dis 1997; 30: 483-8.

28. van Bommel EFH, Hesse CJ, Jutte NHPM, Zietse R, Bruining HA, Weimar W. Impact of continuous hemofiltration on cytokines and cytokine inhibitors in oliguric patients suffering from systemic inflammatory response syndrome. Renal Failure 1997; 19 (3): 443-54.

29. Ronco C, Tetta C, Lupi A, et al. Removal of platelet-activating factor in experimental continuous arteriovenous hemofiltration. Crit Care Med 1995; 23: 99-107.

30. Gotloib L. Hemofiltration in multiorgan failure syndrom secondary to sepsis: A critical analysis of heterogeneity. Nephron 1996; 73: 125-30. 